\title{
Quantitative Inspection of Coatings Thickness by Time-Power Transformation Flash Pulse Thermographic Method ${ }^{\dagger}$
}

\author{
Michal Švantner *, Lukáš Muzika and Šárka Houdková \\ New Technologies - Research Centre, University of West Bohemia, 30100 Plzeň 3, Czechia; \\ muzika@ntc.zcu.cz (L.M.); houdkov@ntc.zcu.cz (Š.H.) \\ * Correspondence: msvantne@ntc.zcu.cz; Tel.: +420-737-914-978 \\ + Presented at the 15th International Workshop on Advanced Infrared Technology and Applications \\ (AITA 2019), Florence, Italy, 17-19 September 2019.
}

Published: 25 September 2019

\begin{abstract}
Thermographic testing is an inspection method, which primary indicates a presence of discontinuities in a tested sample. Its application to coatings can indicate a presence of local thickness variations; however, it mostly does not bring a quantitative information about a thickness of the coatings. This contribution is focused on a quantification of the thermographic inspection, which would make possible an evaluation of coating thickness differences. A flash pulse thermographic testing was applied to thermally sprayed coatings. An importance of a precise synchronization of a flash-source and thermographic recording was determined. Different evaluation methods were analyzed and their comparison showed that a time-power transformation method is the most suitable for a quantification of the inspection results.
\end{abstract}

Keywords: thermographic testing; coatings; infrared non-destructive testing; flash-pulse thermography

\section{Introduction}

Thermographic testing [1], which is also called infrared non-destructive testing (IRNDT), is an active thermography method, which is used for a near-surface material inspection. The method is based on an external excitation, which induces a thermal process inside the inspected object. Material inhomogeneities near a tested surface affect the process and can be detected by a measurement of a thermal response of the object. A contrast of small spatial differences of the thermal response (indications) are mostly increased using special data processing methods. The indications can be connected with defects or inhomogeneities in the tested object. There was developed a number of IRNDT methods, which used different excitation procedures and evaluation methods. An overview of thermographic testing can be found in [2], state-of-the-art and trends of IRNDT can be found in [3].

A benefit of the thermographic methods is that they allow an area inspection. The indications mostly bring an information about an occurrence and position of inhomogeneities without any additional quantification of the results, as for example a depth of found defects or a thickness of inspected coatings. Thus, extended evaluation methods have been developed for a quantification of IRNDT results. To these methods belong, for example, an evaluation of Contrast to Noise Ratio [4] or Probability of Detection [5] methods. These methods, however, allow a quantification of an intensity of the indications only. Thus, they are mostly useful for an evaluation of a detectability, but they are not usable for a quantitative IRNDT inspection.

The research in contribution was focused on a quantitative evaluation of a coating thickness by a flash pulse thermography. The pulse thermography is based on an excitation by a short or long pulse using flash lamps, a laser or halogen lamps. A thermal response of an excited object is mostly 
evaluated by one of the standard methods, which are, for example, signal derivation, pulse-phase evaluation or thermographic signal reconstruction. The pulse thermography is introduced more in detail, for example, in publications [6] or [7]. The flash pulse thermography is one of the thermographic methods, which can be very efficient for an inspection of coatings quality, as it was presented for example in [8] or [9]. Advanced procedures for a quantitative evaluation of a coatings thickness were introduced in [10]. These procedures were based on a comparison of an experimental measurement with an analytical model. A method of a quantitative evaluation of pulse and lock-in thermography based on a correlation of Fast Fourier transform results with a real thickness of inspected coatings was presented in [11]. Similar approach was used in this research, where the correlation of IRNDT experimental results with a real thickness of tested coatings was done. Three IRNDT evaluation method were applied on measured flash pulse excitation response and the results were compared with each other and with unprocessed thermographic signals. An influence of a precision of a synchronization of a thermographic camera and the flash pulse was also analyzed. This factor appeared to be one of the most significant for a repeatable and repeatable quantitative measurement.

\section{Experiment description}

The topic of this research was a quantitative investigation of a thickness of thermally sprayed coatings by a flash pulse thermographic inspection and different evaluation methods. The experimental samples were $\mathrm{Cr} 13 \%$ Fe coatings, which were made on steel substrates by a Twin Wire Arc Spraying (TWAS) technology [12]. Coatings of the thickness $0.22,0.38,0.49$ and $0.59 \mathrm{~mm}$ were deposited on the steel substrate of a thickness about $5 \mathrm{~mm}$. An area of the each sample was about $25 \times 20 \mathrm{~mm}$. The surface of the coatings were left in the as-sprayed state, without any subsequent treatment.

Each sample was inspected using the same testing configuration. A flash lamp with a pulse length about $6 \mathrm{~ms}$ and the maximum power $6 \mathrm{~kJ}$ was used for an excitation. A thermal response was recorded by a high-speed cooled InSb based detector thermographic camera with a sensitivity about $20 \mathrm{mK}$ and an integration time $1.4 \mathrm{~ms}$. A distance between the flash lamp and the samples was about $500 \mathrm{~mm}$. The infrared camera framerate was $250 \mathrm{~Hz}$. A synchronization between the flash lamp and the camera was realized by a hardware unit, which started a recording and initiated a flash pulse. The camera recorded both the pulse and the response. One thousand frames were taken from a first frame after the excitation for a subsequent evaluation. Three evaluation methods were applied: FFT based processing (phase evaluation), time-derivation based processing and a P-function method. The time-derivation method was based on an approximation of a temperature time curve in each pixel by a suitable polynomial function and a performing of $1^{\text {st }}$ time-derivative of this function (TDTS - Time Derivation of Thermographic Signal [4]). The P-function method is based on a time-power transformation of the thermographic signal in each pixel, where a position of a minimum point of this transformed function on a time axis is an evaluated quantity. Principles of this method are described, for example, in [13]. A maximum difference, i.e. maximum contrast, between the coatings of different thickness was then found for each evaluation method. The results were compared with each other and with an unprocessed data (i.e., temperature measurement) as well.

\section{Results}

The very first experiments showed an insufficient repeatability of the thermographic measurement of a response after a flash pulse. Phase shifts were compared and differences of about $20 \%$ between results of repeated measurements of the same sample at the same conditions were found. Such a differences were comparable with differences caused by a change of a thickness of the tested coatings. It was revealed that this instability was related to a synchronization of a flash pulse and a thermographic data recording.

A length of the flash pulse was about $6 \mathrm{~ms}$, the infrared camera integration time was $1.4 \mathrm{~ms}$ and the framerate was $250 \mathrm{~Hz}$, that means the frames were recorded with the period of $4 \mathrm{~ms}$. It was revealed that the flash pulse peak can occur at different positions between two recorded subsequent frames. This uncertainty did not affect significantly a standard qualitative defects detection 
procedure, where local inhomogeneities were to be found. However, it was unacceptable for a repeatable quantitative evaluation of differences between the thicknesses of the coatings. Thus, changes of the experimental configuration related to a synchronization of the flash lamp and the infrared camera were made, which increased a repeatability of the measurement. The result of these changes was that the uncertainty of a position of the flash pulse peak in a relation to the recorded frames was much lower and the resulting average difference of results of the repeated measurements was about $1.2 \%$. Such repeatability was already sufficient.

Results of the different evaluation methods brought different numerical values of the evaluated quantities. The dependence of these quantities on the coatings thickness were therefore not comparable with each other. Thus, dependences of relative change of the quantities with respect to the coating of thickness $0.22 \mathrm{~mm}$ were expressed and showed in Figure 1.

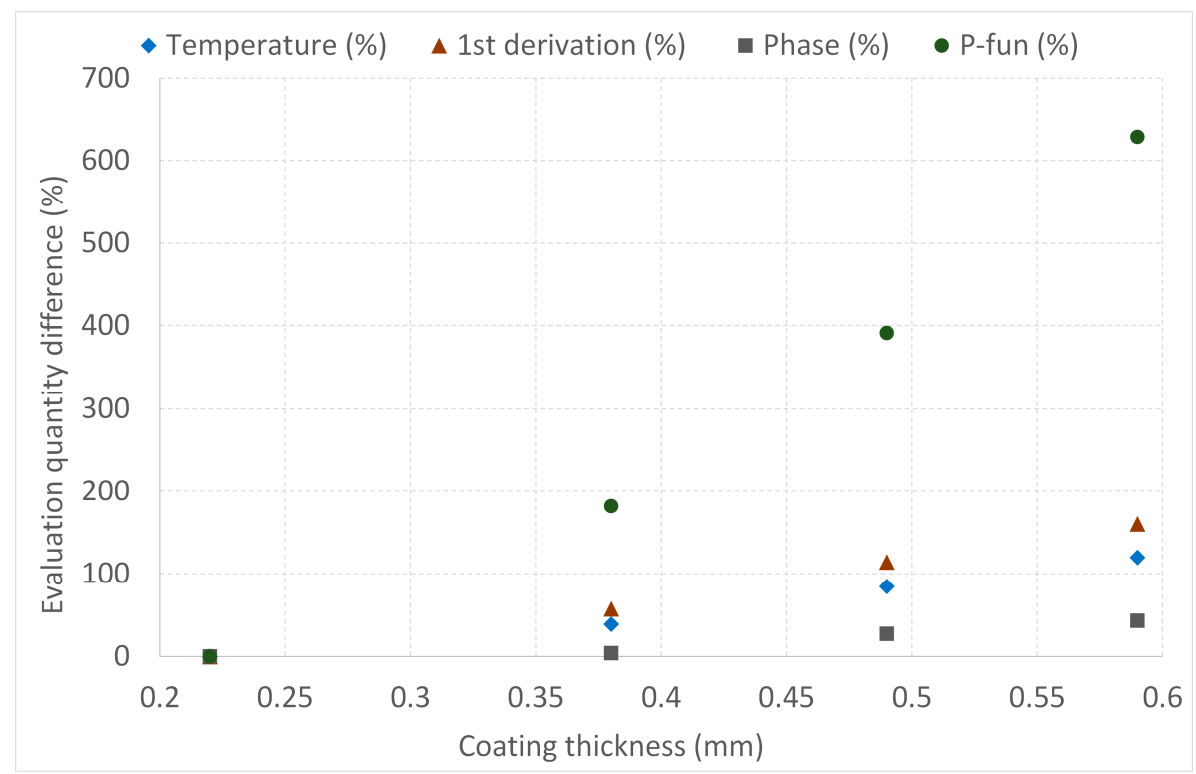

Figure 1. Dependence of IRNDT evaluation results obtained by different methods on coating thickness.

A sensitivity of the methods, which means a ratio of a change of their results to a change of a coating thickness, is the most important indicator of their suitability. The performed experiment showed that the sensitivity of the tested methods to a change of the coating thickness of about $0.1 \mathrm{~mm}$ is in a range from a few percent to about $200 \%$. The best results brought the P-function method, which showed an increase of an evaluation quantity of about $600 \%$ when the coating thickness increased from 0.22 to $0.59 \mathrm{~mm}$. The increase of the $1^{\text {st }}$ derivation method and of the unprocessed thermographic signal was 160 and $120 \%$, respectively. The worst results were obtained by the FFT phase shift evaluation, at which the increase was about $40 \%$ only. This method therefore appeared to be the least appropriate for a quantitative evaluation of the coating thickness.

A linearity of a dependence of the measurement results on the thickness is the second important parameter. None of the methods compared provided a fully linear dependence. However, a very big sensitivity of the P-function method to the coating thickness changes made this method a favorite one for a quantitative evaluation of the thickness of thermally sprayed coatings.

\section{Conclusions}

The flash pulse thermographic method for quantitative evaluation of coating thickness based on a correlation of evaluation results with the real coating thickness was introduced in this contribution. That is not a physical model approach and the presented results are therefore dependent on an experimental setup configuration and coatings physical properties. The obtained numerical values are valid for the tested TWAS Cr 13\% Fe coatings and an experimental correlation should be done for 
different materials, different measurement configurations and also for different ranges of coatings thicknesses. However, especially a sensitivity of the P-function evaluation, which is about $17 \%$ per $10 \mathrm{um}$ of the coating thickness, was very good. The method thus can be considered for a quantitative estimation of coating thickness differences after an adequate calibration.

The results of the research also show a critical influence of a synchronization of a pulse excitation and a thermographic recording. This issue could be solved by a significant increase of a recording frequency (framerate), which would provide a better determined position of a flash peak in relation to individual recorded frames. However, an acceptable repeatability, which is about $1.2 \%$ in our experiments, can be obtained using a flash lamp if a suitable hardware/software configuration is used.

Funding: The work was supported by ERDF project "LABIR-PAV/Pre-application research of infrared technologies" Reg. No. CZ.02.1.01/0.0/0.0/18_069/0010018.

Conflicts of Interest: The authors declare no conflict of interest.

\section{References}

1. Maldague, X.P. V. Theory and practice of infrared technology for nondestructive testing; Wiley series in microwave and optical engineering. Wiley: New York, USA, 2001; ISBN 9780471181903.

2. Usamentiaga, R.; Venegas, P.; Guerediaga, J.; Vega, L.; Molleda, J.; Bulnes, F.G. Infrared Thermography for Temperature Measurement and Non-Destructive Testing. Sensors 2014, 14, 12305-12348.

3. Vavilov, V. Thermal NDT: historical milestones, state-of-the-art and trends. Quant. Infrared Thermogr. J. 2014, 11, 66-83.

4. Švantner, M.; Muzika, L.; Chmelík, T.; Skála, J. Quantitative evaluation of active thermography using contrast-to-noise ratio. Appl. Opt. 2018, 57, D49-D55.

5. Junyan, L.; Yang, L.; Fei, W.; Yang, W. Study on probability of detection (POD) determination using lockin thermography for nondestructive inspection (NDI) of CFRP composite materials. Infrared Phys. Technol. 2015, 71, 448-456.

6. Balageas, D.L. Defense and illustration of time-resolved pulsed thermography for NDE. QIRT J. 2012, 9, 332.

7. Maldague, X.; Galmiche, F.; Ziadi, A. Advances in pulsed phase thermography. Infrared Phys. Technol. 2002, $43,175-181$.

8. Ptaszek, G.; Cawley, P.; Almond, D.; Pickering, S. Transient thermography testing of unpainted thermal barrier coating (TBC) systems. NDT E Int. 2013, 59, 48-56.

9. Zhao, S.; Zhang, C.; Wu, N.; Wang, H. Quality evaluation for air plasma spray thermal barrier coatings with pulsed thermography. Prog. Nat. Sci. Mater. Int. 2011, 21, 301-306.

10. Altenburg, S.J.; Krankenhagen, R.; Bavendiek, F. Thickness determination of polymeric multilayer surface protection systems for concrete by means of pulse thermography. AIP Conf. Proc. 2017, 1806.

11. Shrestha, R.; Kim, W. Evaluation of coating thickness by thermal wave imaging: A comparative study of pulsed and lock-in infrared thermography - Part II: Experimental investigation. Infrared Phys. Technol. 2018, 92, 24-29.

12. Arizmendi-Morquecho, A.; Campa-Castilla, A.; Leyva-Porras, C.; Aguilar Martinez, J.A.; Vargas Gutiérrez, G.; Moreno Bello, K.J;; López López, L. Microstructural Characterization and Wear Properties of Fe-Based Amorphous-Crystalline Coating Deposited by Twin Wire Arc Spraying. Adv. Mater. Sci. Eng. 2014, 2014, 11.

13. Vavilov, V.P.; Pawar, S.S. A novel approach for one-sided thermal nondestructive testing of composites by using infrared thermography. Polym. Test. 2015, 44, 224-233.

(C) 2019 by the authors. Licensee MDPI, Basel, Switzerland. This article is an open access article distributed under the terms and conditions of the Creative Commons Attribution (CC BY) license (http://creativecommons.org/licenses/by/4.0/). 